\title{
A Theory of Entangled Polymers
}

\author{
Yoshiaki CHIKAHISA and Toshiro MorooKA* \\ Laboratory of Physics, Faculty of General Education, \\ Tokyo University of Agriculture and Technology, \\ Fuchu-shi, Tokyo 183, Japan.
}

(Received April 18, 1978)

\begin{abstract}
A molecular theory relating to entanglement is presented herein. The concept of our model is similar to that of Chompff, et al. Since only long relaxation times are important, a light elastic string model is used instead of the Rouse chain. The molecular string is immersed in a viscous medium, and also, due to entanglements, $\delta$ function-like frictional forces are exerted on $p$ points randomly distributed along the string. An exact characteristic equation for relaxation times is obtained. Relaxation spectra in cases where $p=$ 2, 4, 6 and 8, are computed numerically. It is shown that the spectrum consists of three parts: a short time region of the wedge type having a slope, $-1 / 2$, a middle time region of the flat type, and a long time region having a hump. Also, the maximum relaxation time is proportional to $M^{3}$ if the parameter of the enhanced friction at an entangled point is independent of $M$, where $M$ is the molecular weight of polymer.

KEY WORDS String Model / Entangled Points / Random Distribution /

Relaxation Times / Characteristic Equation / Relaxation Spectra /
\end{abstract}

So far there have been many theoretical investigations on entanglement effects in melt or concentrated polymers. Work up to 1973 was carefully reviewed by Graessley. ${ }^{1}$ Recent topological considerations ${ }^{2-4}$ should be noted. However, the essence of entanglement is still obscure. All we can do at the present stage is the examination of the influence of each individual effect relating to the entanglements on viscoelasticities.

Ferry, et al., ${ }^{5}$ proposed a modified Rouse model to elucidate viscoelastic properties of entangled systems. Afterward, Chompff and Duiser, ${ }^{6}$ Chompff and Prins ${ }^{7}$ developed molecular theories of entanglement networks. In their theories, they have used the relaxation spectrum $H(p)$ of a chain with $p$ "slow points" (or "entangled points"), at which the enhanced local friction is assumed to act owing to entanglement. In order to calculate $H(p)$, however, they applied an approximate treatment introduced by Kronig and Penney. ${ }^{8}$ Recently, Hansen, et al., ${ }^{9}$ and Hong, et al., ${ }^{10}$ treated a similar

\footnotetext{
* Present Address: Wood Research Institute, Kyoto University, Uji-shi, Kyoto 611, Japan.
}

modified Rouse model containing entangled points with increasing friction coefficients toward the interior of the chain, where the elastic interactions between entangled points are taken account. However, the entangled points are assumed to be located at regular intervals along the chain; justification of the elastic interactions is not clear.

In this paper, it is assumed that the entangled points distribute randomly along the chain. A light elastic string model is used instead of the Rouse model (the bead and spring model). The reason is that entanglement effects are dominant in very slow time- and at low frequence-regions of dynamics. The hydrodynamic interaction and the excluded volume effect may be neglected in concentrated polymer systems. For simplicity, the mico-Brownian motion of the string is not taken into account. Let the number of entangled points in a string be $p$, which will be proportional to the contor length of the string and hence to the molecular weight, $M$, of the coiled chain polymer. Then an exact characteristic equation for relaxation times, a transcendental equation, can be obtained. For cases where $p=2,4,6$ and 8 , 


\section{Y. ChIKAHISA and T. MorookA}

the equations for various sets of distribution of entangled points are solved numerically by electronic computers, and the relaxation spectra in a long time region are determined. Moreover, it is shown that the maximum relaxation time is proportional to $M^{3}$ if an enhanced friction parameter $\rho$ defined below is independent of $M$.

\section{NO ENTANGLEMENT}

First we discuss the case of no entangled points, using the string model. A light elastic string is suspended in a viscous medium whose friction constant per unit length of the string is $\zeta_{0}$. The inertia effect of the string is neglected. The position of each part of the string is measured along the string when in the equilibrium state. For the displacement $W(x, t)$ from the equilibrium at position $x$ of the string and at time $t$, the fundamental equation is given as,

$$
\frac{c}{L^{2}}-\frac{\partial^{2} W}{\partial x^{2}}=\zeta_{0} \frac{\partial W}{\partial t}
$$

where $c$ is the elastic constant, $2 L$ is the length of the string in equilibrium. In this equation, $x$ expresses the coordinate along the string when its total length $2 L$ is normalized as 2 , and hence the variable range of $x$ is between 1 and -1 . We need normal modes in the form

$$
W(x, t)=w(x) \exp (-t / \tau)
$$

Substitution of eq 2 into eq 1 yields

$$
w^{\prime \prime}+k^{2} w=0
$$

where the dimensionless quantity $k$ corresponds to a wave number in the vibration problem of a string, and the relaxation time $\tau$ is given by

$$
\tau=\zeta_{0} L^{2} / c k^{2}
$$

Under the following boundary condition

$$
w^{\prime}(-1)=w^{\prime}(+1)=0
$$

we easily obtain

$$
k_{n}=n \pi / 2
$$

Hence,

$$
\tau_{n}^{(0)}=\frac{4 \zeta_{0} L^{2}}{\pi^{2} c n^{2}} \quad(n=1,2, \cdots)
$$

where the superscript, 0 means that there is no entangled point, and the subscript $n$ indicates the mode number. The condition (eq 5) means that both ends of the string are free.
Equation 7 agrees with the well-known Rouse result,

$$
\tau_{n}^{(0)}=\frac{b^{2} N^{2} f_{0}}{6 \pi^{2} k_{\mathrm{B}} T n^{2}}
$$

if one uses the following correspondences

$$
\begin{aligned}
& 2 L \leftrightarrow N b \\
& \zeta_{0} \leftrightarrow f_{0} b^{-1} \\
& c \leftrightarrow 6 k_{\mathrm{B}} T b^{-1}
\end{aligned}
$$

where $N$ is the number of segments, $b$ is the root mean square distance of a segment in the Rouse model, $f_{0}$ is the friction constant between a segment and medium, $k_{\mathrm{B}}$ is the Boltzmann constant, and $T$ is the absolute temperature. Figure 1 is the histo-

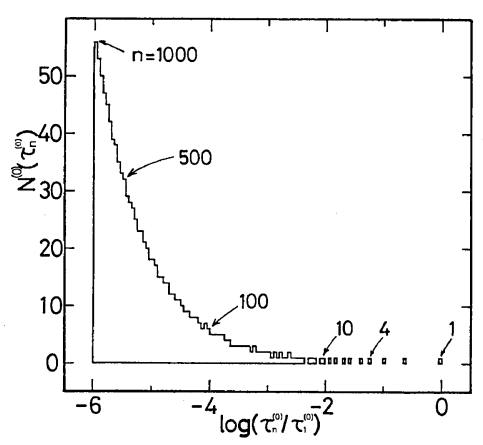

Figure 1. Histogram of relaxation times in the case of no entanglements $(p=0)$. The width of the abscissa, $\Delta$, is 0.05 .

gram of relaxation times from the first to the thousandth mode when the width $\Delta$ of the abscissa is 0.05 . The abscissa is $\log \left(\tau / \tau_{1}^{(0)}\right)$ and the ordinite $N^{0}(\tau)$ is the number of modes of which the relaxation time exists in the range between $\log \left(\tau / \tau_{1}^{(0)}\right)$ and $\log \left(\tau / \tau_{1}^{(0)}\right)+\Delta$. Relaxation times from the 1 st to the 10th mode are discrete, but the those more than the 10th are almcst continuous. In Figure 2, $\log N^{(0)}$ is plotted against $\log \left(\tau / \tau_{1}^{(0)}\right)$. As is well known, the wedge-type spectrum with its slope $-1 / 2$ appears in the short time region. However, it should be noted that in the long time region there are discrete spectra over 2 decades, each of which has a relaxation strength of $\nu k_{\mathrm{B}} T$, where $\nu$ is the number density of polymers.

In slow relaxation phenomena, long relaxation times are important. For example in the case of no entanglement, the steady viscosity $\eta$ is given by, 


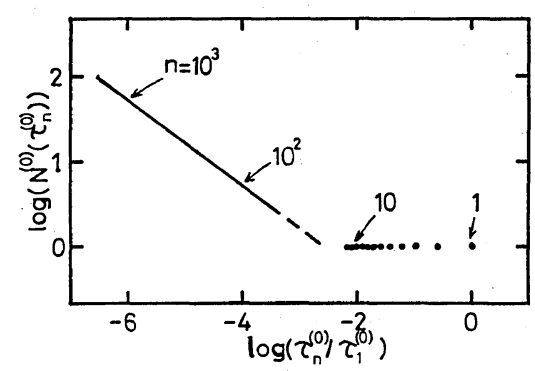

Figure 2. Relaxation spectrum in the case of no entanglements.

$$
\begin{aligned}
& \left(\eta-\eta_{\mathrm{s}}\right) / \nu k_{\mathrm{B}} T=\tau_{1}{ }^{(0)} \sum_{n=1}^{N} n^{-2} \simeq \tau_{1}{ }^{(0)} \sum_{n=1}^{\infty} n^{-2} \\
& \quad=\left(\pi^{2} / 6\right) \tau_{1}{ }^{(0)}
\end{aligned}
$$

where $\eta_{\mathrm{s}}$ is the viscosity of solvent. If this quantity is approximated by

$$
\left(\eta-\eta_{\mathrm{s}}\right) / \nu k_{\mathrm{B}} T \simeq \tau_{1}^{(0)} \sum_{n=1}^{\mu} n^{-2}
$$

each value amounts to $61 \%, 87 \%$, or $94 \%$ against the exact value $\left(\pi^{2} / 6\right) \tau_{1}^{(0)}$, according as $\mu=1,4$ or 10. In order to deal with entanglements, therefore, it is presumably sufficient to take into account the changes in a few modes with long relaxation times.

\section{ENTANGLEMENTS}

Our model for entanglements is as follows. Owing to entanglements, $\delta$ function-like frictional forces act on randomly distributed $p$ points along the string, besides the uniform friction. Thus, instead of eq 1 , the fundamental equation for the displacement $W(x, t)$ is,

$$
\frac{c}{L^{2}} \cdot \frac{\partial^{2} W}{\partial x^{2}}=\zeta_{0}\left\{1+\rho \sum_{j=1}^{p} \delta\left(x-a_{j}\right)\right\} \frac{\partial W}{\partial t}
$$

where $a_{j}$ is the position of the $j$-th entangled point, and $\rho$ is the strength of friction at an entangled point, $a_{j}$ 's are ordered so that $-1 \leq a_{1} \leq \ldots \leq a_{\mathrm{p}}$ $\leq 1$. The simple case of $p=1$ has been discussed already in standard textbooks of mathematical physics. For arbitrary $p$ and $a_{j}$ 's, we seek here normal modes in the form of eq 2 , which yields an equation of the Strum-Liouville type for $w(x)$ :

$$
w^{\prime \prime}+k^{2}\left\{1+\rho \sum_{j=1}^{p} \delta\left(x-a_{j}\right)\right\} w=0
$$

The wave number $k$ is related to the relaxation time $\tau^{(p)}$ by eq 4 . We set

$$
\begin{array}{lr}
w_{1}=N_{1} \cos k x & \text { for }-1 \leq x \leq a_{1} \\
w_{j}=N_{j} \cos k x+M_{j} \sin k x & \text { for } a_{j-1} \leq x \leq a_{j} \\
w_{p+1}=N_{p+1} \cos k x & \text { for } a_{p} \leq x \leq 1
\end{array}
$$

where $j=2,3, \ldots, p$. We proceed to determine the number $k$ so that these functions satisfy the additional boundary conditions, as well as eq 5 ,

$$
\begin{aligned}
& w_{j}\left(a_{j}\right)=w_{j+1}\left(a_{j}\right) \\
& w_{j}\left(a_{j}\right)-w^{\prime}{ }_{j+1}\left(a_{j}\right)=k^{2} \rho w_{j}\left(a_{j}\right)
\end{aligned}
$$

for $j=1,2, \ldots, p$. Equation 16 is obtained by integration of eq 13 over a narrow range of $x$ involving $x=a_{j}$. The characteristic equation for $k$ becomes a $2 p$ dimensional determinant. However, after a lengthy calculation by the use of an inductive method for the number of entangled points $p$, it is proved exactly that the characteristic equation reduces to

$$
D^{(p)}\left(k ; a_{1}, \ldots, a_{p}, \rho\right)=0
$$

with

$$
D^{(p)}=\sin 2 k+\sum_{n=1}^{p}(-1)^{n-1}(\rho k)^{n} \Xi_{n}
$$

where

$$
\begin{aligned}
\Xi_{n} & =\sum_{1 \leq i_{1}<\cdots<i_{n} \leq p} \sum \ldots \cdots \sum \cos k\left(1+a_{i_{1}}\right) \\
& \times\left[\prod_{m=2}^{n} \sin k\left(a_{i_{m}}-a_{i_{m-1}}\right)\right] \cos k\left(1-a_{i_{n}}\right)
\end{aligned}
$$

The construction of eq 18 and 19 is interpreted as follows. The first term $\sin 2 k$ is the contribution from a free string with no entangled point. Each term involving $\rho^{n}$ indicates a motion in which the string has all $n$ effective entangled points of the $p$ ones, and that the number of such terms in $\Xi_{n}$ is equal to the combination number ${ }_{p} C_{n}$. Especially, the term $\rho^{p}$ indicates that all $p$ entangled points are effective. These situations are expressed in Figure 3 in which $\otimes$ means an effective entangled point, and $\times$, a non-effective one.

Equations 18 and 19 are rewritten in simpler form using matrices. Let us introduce a row vector $\boldsymbol{C}$, a column vector $\boldsymbol{G}$ and a square matrix $\boldsymbol{S}$ as follows:

$$
C=\left(C_{1}, \ldots, C_{p}\right), G^{\mathrm{T}}=\left(G_{1}, \ldots, G_{p}\right)
$$

where,

$$
C_{i}=\cos k\left(1+a_{i}\right), G_{i}=\cos k\left(1-a_{i}\right)
$$

The $i j$-th element $S_{i j}$ of $S$ is given by

$$
S_{i j}= \begin{cases}\sin k\left(a_{j}-a_{i}\right) & \text { for } i<j \\ 0 & \text { for } i \geq j\end{cases}
$$




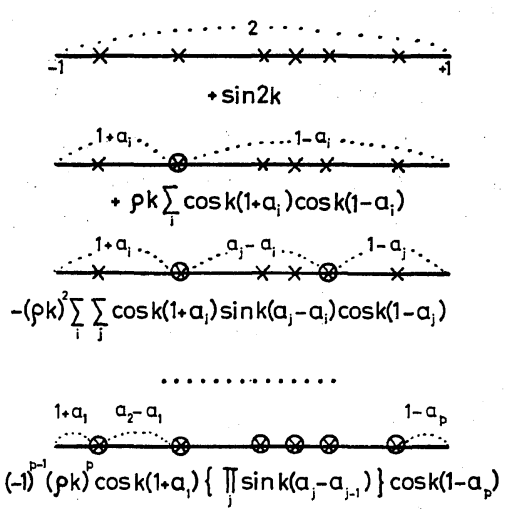

Figure 3. Formulation of the characteristic equation 17. The total length of the string is normalized as 2 . $x$ is a non-effective entangled point, and $\otimes$ is an effective entangled point.

where $i, j=1,2, \ldots, p$ and the superscript $\mathrm{T}$ indicates the transposition of the given vector. It is easily shown that eq 18 and 19 yield

$$
D^{(p)}=\sin 2 k+\rho k \boldsymbol{C} \cdot \boldsymbol{D}^{(p)} \cdot \boldsymbol{G}
$$

where the matrix $D^{(p)}$ is

$$
D^{(p)}=\frac{E-(-1)^{p} T^{p}}{E+T}
$$

Here, $\boldsymbol{E}$ is the unit matrix of order $p$, and

$$
\boldsymbol{T}=\rho k \boldsymbol{S}
$$

The numerator of eq 24 has a factor $(\boldsymbol{E}+\boldsymbol{T})$, and hence the matrix $D^{(p)}$ has no singular point. For instance, in the cases of $p=2,4,6$, and 8 , the matrices $\boldsymbol{D}^{(p)}$ 's become, respectively,

$$
\begin{aligned}
& D^{(2)}=E-T, \quad D^{(4)}=(E-T) \cdot\left(E+T^{2}\right) \\
& D^{(6)}=(E-T) \cdot\left(E+T^{2}+T^{4}\right) \\
& D^{(8)}=(E-T) \cdot\left(E+T^{2}\right) \cdot\left(E+T^{4}\right)
\end{aligned}
$$

\section{RELAXATION SPECTRA}

Let the molecular weight, $M$, of the primary polymer be mono-dispersed. The average molecular weight, $M_{\theta}$, between two neighboring entangled points in a polymer molecule is constant and independent of $M$, if $M$ is much larger than $M_{\mathrm{e}}$. Hence it is considered that the number of entangled points per polymer, $p$, is constant for a given $M$, and it holds that $p=M / M_{\mathrm{e}}$. The entangled polymer is considered to be an ensemble of the systems each having a different set of $p$ entangled points distributed randomly. Thus, the physical property observed macroscopically is the ensemble average of the physical quantity.

For fixed values of $p$ and $\rho$, if one selects a set of $a_{1}, \ldots, a_{p}$, a set of relaxation times $\tau_{1}^{(p)}$, $\tau_{2}^{(p)}, \ldots$ is obtained by solving the characteristic equation 17. Here $\tau_{n}{ }^{(p)}$ is the relaxation time when the number of entangled points is $p$ and the mode number corresponding to eq 17 in the case of no entanglement is $n$. For an example, Figure 4 shows the oscillatory behavior of $D^{(6)}(k)$ given by

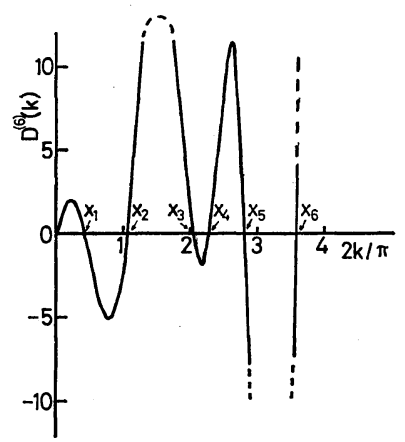

Figure 4. The oscillatory behavior of $D^{(8)}(k)$ given by eq 23 when $p=6, \rho=1.0$ and $a_{i}$ 's take on the values given in the text.

eq 23 when $p=6, \rho=1.0, a_{1}=-0.710719, a_{2}=$ $-0.674269, a_{3}=-0.577361, a_{4}=-0.133809, a_{5}=$ $0.705250, a_{6}=0.854109$. The cross-points with the abscissa give the roots $x_{1}, x_{2}, \cdots, x_{n}, \cdots$ of eq 17 , and hence the ratios of the respective relaxation times $\tau_{n}{ }^{(0)}$ to $\tau_{1}{ }^{(0)}$ are determined, using eq 4 and 7. If another set of $\left\{a_{j}\right\}$ is selected, another set of $\left\{\tau_{n}^{(p)}\right\}$ is obtained. It is expected, therefore, that the original discrete distribution of relaxation times in the long time region of Figures 1 and 2 changes to a continuous distribution when $a_{j}$ 's are randomly selected.

Figures 5, 6, and 7 are the relaxation time spectra obtained numerically by computer for various $\rho$ 's values when $p=2,4,6$, and 8 . Figure 5 is the spectrum of relaxation times in the range from the first to the hundredth mode for $p=6, \rho=1.0$ when 128 set of $\left\{a_{j}\right\}$ are randomly selected. As is expected, the spectrum more than about the 7 th mode is the wedge type with a slope of $-1 / 2$. This indicates that the relaxation times in this region are not influenced by the introduction of entangled points. However, the discrete spectrum from the 1st to the 10th mode in Figures 1 and 2 


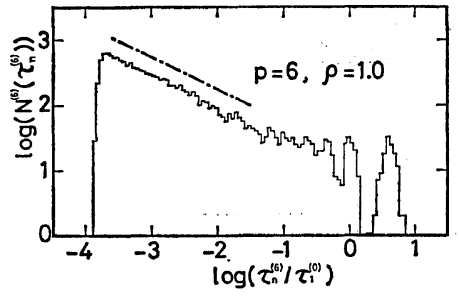

Figure 5. Relaxation spectrum for the range from the 1 st to the 100 th mode when $p=6$ and $\rho=1.0$. Number of sets of random $\left\{a_{i}\right\}$ is 128 , and $\Delta=0.05$.

changes to a continuous one in Figure 5. Further, it should be noted that the 1st mode in Figure 5 is isolated and makes a hump. That is, long relaxation times are influenced by the existance of entangled points. Figures 6 and 7 show the histograms from the 1st to the 4th mode for various values of $p$ and $\rho$, in which 512 sets of $\left\{a_{j}\right\}$ are randomly selected. The values of $p$ are $2,4,6$ and 8 , and $\rho$ are $0.4,1.6,6.4$ and 25.6. The ordinates in Figures 6 and 7 are the ratios of frequencies $f^{(p)}\left(\tau^{(p)}\right)$ to 512 , where $f^{(p)}\left(\tau^{(p)}\right)$ is the number of the roots of the characteristic equation 17 existing between $\log \left[\tau^{(p)} / \tau_{1}^{0}\right]$ and $\log \left[\tau^{(p)} / \tau_{1}^{(p)}\right]+\Delta$, where $\Delta$ is 0.05 . Thus $100 \%$ indicates a sharp line spectrum with a relaxation strength $\nu k_{\mathrm{B}} T$. In the figures, the arrows indicate maximum relaxation times. For small $\rho$ 's each spectrum is separated. With increasing $\rho$, there occurs an overlap in the $2 \mathrm{nd}, 3 \mathrm{rd}$, and 4 th mode. However, the 1st mode is rather isolated; that is, it makes a hump. When $p$ is increased for a fixed $\rho$, the over-all shape of the spectra hardly changed but a shift to the longer time region occurs.

In the computations mentioned above, it was found that, for fixed $p$ and $\rho$, maximum relaxation times $\tau_{\max }^{(p)}$ appears when $a_{1}=a_{2}=\cdots=a_{p / 2}=-1$ and $a_{p / 2+1}=\cdots=a_{p}=+1$. Here $p$ is assumed to be an even number. Then eq 17, 18, and 19 reduce simply to

$$
p \rho k \tan k=2
$$

Figure 8 shows the relation between $\log \left[\tau_{\text {max }}^{(p)} / \tau_{1}^{0}\right]$ and $\log p$, obtained by solving eq 27 numerically. From this figure, it is found that $\tau_{\max }^{(p)} / \tau_{1}^{(0)}$ is nearly proportional to $p$. Since $p$ is proportional to the
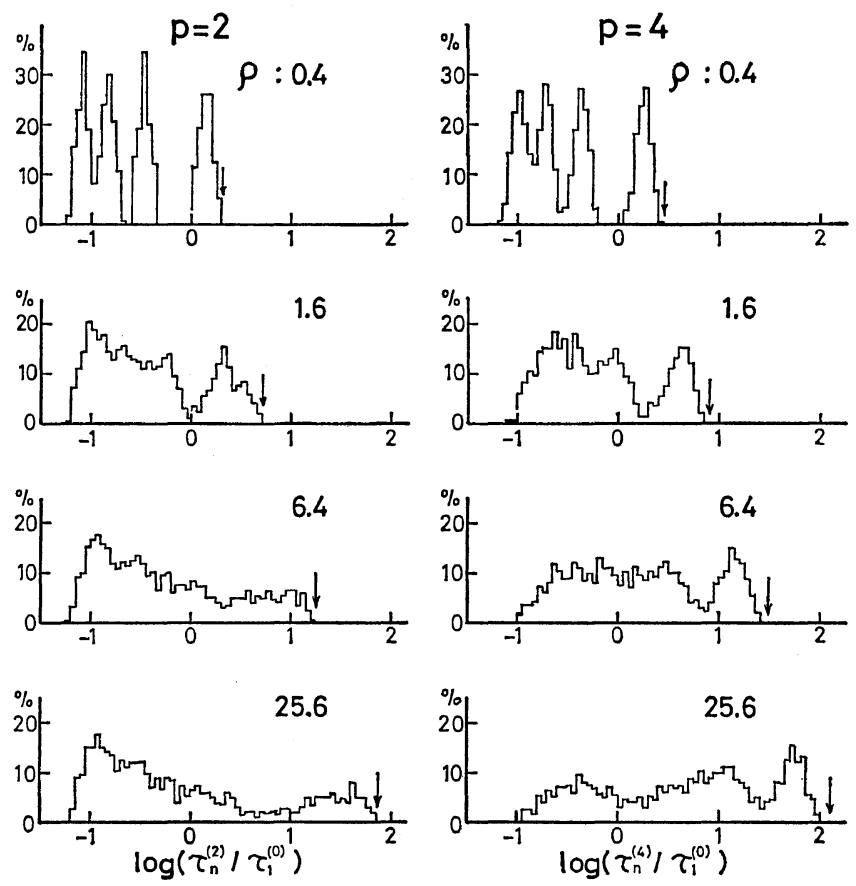

Figure 6. Histograms of relaxation times from the 1st to the 4th mode when $p=2$ and $4 . \quad \rho$ 's values are indicated in the figure. The number of sets of random $\left\{a_{i}\right\}$ is 512 , and $\Delta=0.05$. The arrows indicate the maximum relaxation times for the given $p$ and $\rho$. 


\section{Y. Chikahisa and T. Morooka}
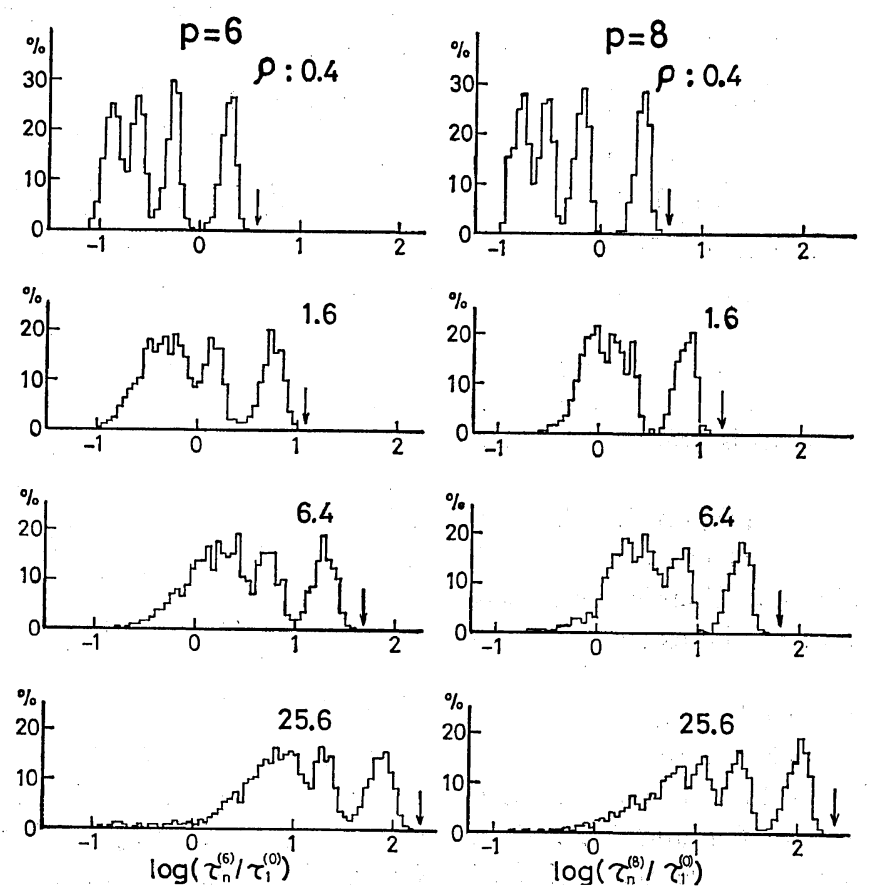

Figure 7. Histograms of relaxation times from the 1 st to the 4 th mode when $p=6$ and 8 . $\rho$ 's values are indicated in the figure. The number of sets of random $\left\{a_{i}\right\}$ is 512 , and $\Delta=0.05$. The arrows indicate the maximum relaxation times for the given $p$ and $\rho$.

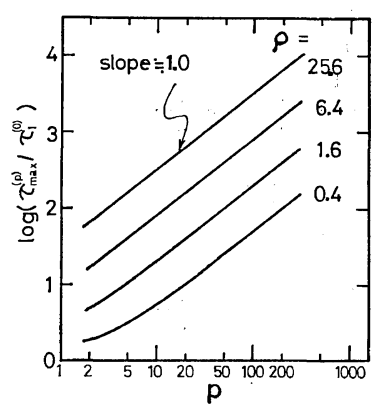

Figure 8. The relation between $\log p$ and $\log \left[\tau_{\max }^{(p)}\right]$ $\left.\tau_{1}{ }^{(0)}\right]$, for the given $\rho$ 's values, where $p$ is the number of entangled points and $\tau_{\max }^{(p)}$ is the maximum relaxation time.

total contor length $2 L$ and hence to molecular weight $M$, using $\tau_{1}{ }^{(0)} \propto L^{2} \propto M^{2}$, we get

$$
\tau_{\max }^{(p)} \propto L^{3} \propto M^{3}
$$

if $\rho$ is constant.

\section{DISCUSSION}

From Figures 5, 6 and 7 it is suggested that the relaxation spectrum of our model, in $\log H v s$. $\log \tau$, consists of the following three parts: a short time region of a wedge type with a slope $-1 / 2$, a middle time region of the flat type coming from several lower order modes except the 1st mode, and a long time region having a hump coming from the 1 st mode. This result agrees qualitatively with experiments by Onogi, et al. ${ }^{11}$ However, our spectrum is different from those predicted theoretically by Chompff, ${ }^{3,4}$ Hansen, ${ }^{6}$ and Hong, et al. ${ }^{7} \quad$ This is due to differences in the models and their approximate calculations.

The result of eq $28, \tau_{\max }=A M^{3}$, agrees with theoretical predictions obtained by one of our group, ${ }^{12}$ de Gennes ${ }^{2}$ and Edwards, ${ }^{3}$ although the respective molecular concepts differ from each other. However, this prediction of $M^{3}$ differs from $M^{3.4}$ as suggested by the mechanical data. This fact suggests that the friction parameter $\rho$ is not constant but increases with an increase of $M$.

Quantitative comparisons with experiments such as viscosity, mechanical modulus, storage compliance, relaxation spectrum will be done in the 


\section{A Theory of Entangled Polymers}

near future. Still, the model and the calculation mentioned above will be applied also to branched polymer systems.

Acknowledgment. The authors wish to thank the late Professor H. Morimoto for his many valuable comments throughout this work. The numerical computations were carried out using the HITAC $8800 / 8700$ of the University of Tokyo and NOVA 01 of Tokyo University of Agriculture and Technology.

\section{REFERENCES}

1. W. W. Graessley, Adv. Polym. Sci., 16, 1 (1974).

2. P. G. de Gennes, J. Chem. Phys., 55, 572 (1971).

3. S. F. Edwards and J. W. V. Grant, J. Phys. A:
Math. Nucl. Gen., 6, 1169 and 1186 (1973).

4. M. Doi, J. Phys. A: Math. Gen., 8, 417 (1975).

5. J. D. Ferry, R. F. Landel, and M. L. Wiliams, J. Appl. Phys., 26, 359 (1955).

6. A. J. Chompff and J. A. Duiser, J. Chem. Phys., 45, 1505 (1966).

7. A. J. Chompff and W. Prins, J. Chem. Phys., 48, 235 (1968).

8. R. de L. Kronig and W. G. Penney, Proc. Roy. Soc. (London), A130, 499 (1931).

9. D. R. Hansen, M. C. Williams, and M. Shen, Macromolecules, 9, 345 (1976).

10. S. D. Hong, D. R. Hansen, M. C. Williams, and M. Shen, J. Polym. Sci., 15, 1869 (1977).

11. S. Onogi, T. Masuda, and K. Kitagawa, Macromolecules, 3, 109 (1970).

12. Y. Chikahisa, J. Phys. Soc. Jpn., 19, 92 (1964). 